\title{
Diversité de la résistance des spores de Bacillus à l'eau oxygénée
}

\author{
par \\ O. CERF et J. HERMIER \\ avec la collaboration technique de F. METRO \\ Station Centrale de Recherches Laitières et de Technologie des Produits Animaux, \\ Institut National de la Recherche Agronomique, \\ Domaine de Vilvert - 78 - Jouy-en-Josas (France)
}

\section{Introduction}

Parmi les appareillages qui sont actuellement commercialisés pour le conditionnement aseptique du lait stérilisé à ultra-haute température, dit lait U.H.T., plusieurs utilisent l'eau oxygénée pour stériliser le matériau d'emballage (Heiss, in Lubieniecki-von Schelborn, 1970 ; Hsu, 1970 ; Ashton, 1971). Or si l'action désinfectante de l'eau oxygénée diluée dans l'eau ou le lait à la température ordinaire est connue de longue date (Lück, 1956), il existe peu de publications consacrées plus précisément à la stérilisation par de l'eau oxygénée concentrée à la température ordinaire, et à chaud. Curran, Evans et Leviton (1940) et Swartling et Lindgren (1962 et 1968) sont les seuls à notre connaissance à avoir étudié l'influence de la température et de la concentration d'une solution d'eau oxygénée sur la résistance des spores de plusieurs souches de Bacillus. Ces souches avaient été prises arbitrairement par Curran et al. (1940) et choisies pour leur résistance à l'eau oxygénée par Swartling et Lindgren (1962). D'après les conclusions de ces auteurs, l'action de l'eau oxygénée dans ces conditions obéirait à des lois simples puisque la courbe de survie des spores en fonction du temps de contact est présentée comme linéaire. Or, la courbe de survie des spores soumises à l'action d'un produit chimique est rarement linéaire (nombreux exemples dans Russel, 1971) et en particulier nous avons montré que la courbe de survie des spores de $B$. subtilis var. niger dans l'eau oxygénée ne l'était pas (Cerf et Hermier, à paraître). La question est donc de savoir si l'eau oxygénée représente réellement une exception parmi les désinfectants. C'est pourquoi nous nous sommes proposé de compléter l'étude de la résistance de la spore bactérienne à l'eau oxygénée en déterminant l'influence de deux autres facteurs : le $\mathrm{pH}$ de la solution d'eau oxygénée et la 
nature de la souche utilisée. Nous l'avons fait en nous plaçant dans deux types de conditions proches de la pratique industrielle actuelle du conditionnement aseptique.

\section{1) SOUCHES BACTÉRIENNES}

Vingt-et-une souches ont été utilisées dans ce travail. Deux sont des souches de collection : Bacillus subtilis NCDO 736 et B. subtilis SJ 2 (CNRZ 603, souche isolée du lait).

Les autres souches ont été isolées au laboratoire au cours d'essais de stérilisation du lait dans un stérilisateur pilote U.H.T. Toutes ces souches, qui proviennent de l'eau ont une croissance abondante dans le lait U.H.T. et y provoquent des modifications caractéristiques. Elles ont été identifiées selon les techniques de Smith, Gordon et Clark (1952). Parmi les souches mésophiles, neuf appartiennent à l'espèce $B$. licheniformis, trois à $B$. cereus, deux à $B$. pumilus et une à $B$. subtilis. Les autres souches de Bacillus, dont l'une est mésophile (souches 109) et les trois autres thermophiles, n'ont pu recevoir de nom d'espèce. Les souches 103 et 105 sont thermophiles strictes, contrairement à la souche 104, et s'apparentent à l'espèce $B$. subtilis.

\section{2) Production des SPORES}

Le milieu utilisé pour la préparation des spores est celui de Wang, Scharer et Humphrey (1964). Il est réparti en boîtes de Roux à raison de $250 \mathrm{ml}$ par boîte, inoculé avec une culture de $20 \mathrm{~h}$ en bouillon nutritif et incubé à $30^{\circ} \mathrm{C}$ pour les mésophiles ou à $55^{\circ} \mathrm{C}$ pour les thermophiles. Le taux de sporulation après $48 \mathrm{~h}$ est, suivant les souches, de 95 à 99 p. 100 pour les mésophiles et de 20 à 80 p. 100 pour les thermophiles. Après 4 jours d'incubation, les spores, dont les cellules-mères sont complètement lysées, sont mises en suspension dans l'eau distillée stérile et conservées à $4^{\circ} \mathrm{C}$. Les spores ne reçoivent aucun traitement thermique entre leur préparation et les essais.

\section{3) Trattement Des SPOREs a $80^{\circ} \mathrm{C}$}

Le traitement des spores est effectué à l'intérieur d'un ballon à fond rond muni de trois ouvertures :

- une ouverture supérieure pour le passage de l'axe du moteur d'agitation ; cette ouverture est rendue étanche par un joint liquide ;

- une ouverture latérale bouchée au coton pour l'introduction de la suspension de spores et les prélèvements en cours de traitement ;

- une autre ouverture latérale, bouchée au coton, pour le passage d'un thermomètre.

Le ballon contenant $49 \mathrm{ml}$ de milieu de traitement est placé dans un bain-marie thermostaté maintenant la température du mi- 
lieu à $80^{\circ} \mathrm{C} \pm 0,5^{\circ} \mathrm{C}$. Le milieu est agité par une hélice en verre montée sur l'axe du moteur d'agitation.

Les milieux de traitement ont la composition suivante :

- pour les essais à $\mathrm{pH} \mathrm{2,} \mathrm{le} \mathrm{milieu} \mathrm{de} \mathrm{traitement} \mathrm{est} \mathrm{constitué}$ par une solution de chlorure de potassium 0,01 M (molarité finale) dont le $\mathrm{pH}$ est réglé par addition de $\mathrm{HCl}$ concentré, à laquelle on ajoute, ou non, de l'eau oxygénée à $150 \mathrm{~g} / 1$ (concentration finale);

- pour les autres valeurs de $\mathrm{pH}$, de l'eau oxygénée à $150 \mathrm{~g} / 1$ (concentration finale) est diluée dans une solution tampon obtenue en mélangeant dans les proportions convenables une solution d'acide citrique $0,02 \mathrm{M}$ et une solution de phosphate disodique $0,02 \mathrm{M}$ (molarités finales).

L'ajustement du $\mathrm{pH}$ aux valeurs prévues se fait à la température à laquelle doivent avoir lieu les essais. L'eau oxygénée est titrée après dilution au centième, en milieu acide, par du permanganate de potassium $0,1 \mathrm{~N}$. On a vérifié que sa teneur ne variait pas au cours des essais. L'eau oxygénée employée est un produit Baker, qualité " analyzed reagent ". Les autres produits sont de la qualité " pour analyse ».

Le milieu de traitement est inoculé avec $1 \mathrm{ml}$ d'une suspension de spores de concentration connue. L'agitation du milieu de traitement permet d'obtenir une suspension de spores complètement homogène en moins de $30 \mathrm{~s}$. Les prélèvements de $1 \mathrm{ml}$ sont transférés dans des tubes contenant $9 \mathrm{ml}$ de milieu de dilution maintenus à la température de la glace fondante. Les pipettes utilisées pour les prélèvements ayant été réchauffées à environ $80^{\circ} \mathrm{C}$, la durée du traitement s'entend jusqu'au moment où le contenu de la pipette est soufflé dans le milieu de dilution.

Le milieu de dilution est une solution à $0,9 \mathrm{~g} / 1$ de chlorure de sodium répartie à raison de $8,5 \mathrm{ml}$ par tube, à laquelle on ajoute $0,2 \mathrm{ml}$ d'une solution à $10 \mathrm{mg} / \mathrm{ml}$ de catalase (Swartling et Lindgren, 1968) (catalase lyophilisée à 3000 U.I./mg, Nutritional Biochemicals Corporation) et $0,3 \mathrm{ml}$ de tampon citrate-phosphate $0,2 \mathrm{M}$ de façon à obtenir un $\mathrm{pH}$ final proche de la neutralité. La solution de catalase est stérilisée par filtration sur une membrane à pores de $0,2 \mu \mathrm{m}$ (Millipore). Quand le milieu de traitement ne contient pas d'eau oxygénée, le milieu de dilution est de l'eau distillée.

\section{4) Traitement des spores a $26^{\circ} \mathrm{C}$}

Le traitement des spores est effectué dans un tube à essais dont le contenu est agité sur un vortex à intervalles de $5 \mathrm{mn}$. Le milieu de traitement est constitué par de l'eau oxygénée à $230 \mathrm{~g} / 1$ (concentration finale), dont le $\mathrm{pH}$ est réglé par dilution dans une solution tampon citrate-phosphate, comme décrit dans le paragraphe précédent. Les prélèvements sont effectués de la même façon que pour 
les spores chauffés à $80^{\circ} \mathrm{C}$, mais les pipettes sont à la température ambiante.

\section{5) DÉNOMBREMENT DES SPORES TRAITÉES}

Après refroidissement et action de la catalase ( $3 \mathrm{mn}$ suffisent), les dilutions sont effectuées dans l'eau distillée, et les numérations faites en double dans des boîtes de Petri contenant du milieu de Wang et al. (1964) solidifié avec $15 \mathrm{~g} / 1$ de gélose. Les boîtes sont incubées à $37^{\circ} \mathrm{C}$ pour les souches mésophiles et à $55^{\circ} \mathrm{C}$ pour les souches thermophiles, pendant $48 \mathrm{~h}$. On a vérifié que l'inoculum n'apportait pas de substances inhibant le développement des spores.

\section{6) Présentation des résultats}

Les courbes de survie obtenues (logarithme de la concentration en spores survivantes en fonction du temps) sont rarement des droites sur toute leur longueur comme cela serait le cas si la destruction des spores pouvait être assimilée à une réaction de premier ordre. Aussi, pour en faire une description simple et complète, nous avons adopté la présentation recommandée par Pflug et Schmidt (1968), mais avec les symboles portés sur les figures 1 et 2 . Lorsque les courbes étaient concaves vers le haut pendant plusieurs réductions décimales (type E, non décrit par Pflug et Schmidt, 1968), et, pour les traitements en présence d'eau oxygénée pour les courbes de type $\mathrm{B}, \mathrm{C}$ et $\mathrm{D}$, les temps nécessaires pour obtenir réellement 4 réductions décimales ont été comparés aux valeurs calculées 4D ou 4Di (pour comparaison avec les valeurs présentées par Swartling et Lindgren, 1968). D est le temps de réduction décimale, c'est-à-dire le temps nécessaire pour réduire de 90 p. 100 la concentration de spores viables ; il est calculé sur une portion rectiligne de la courbe de survie ; pour les courbes de type E, il est calculé pour les 30 premières secondes de traitement.

\section{Résultats}

\section{1) Résistance dans L'eau oXygÉnée a pH 2, a $80^{\circ} \mathrm{C}$}

\section{a) B. subtilis NCDO 736}

La courbe de survie observée avec cette souche présente une traînée à partir de la troisième réduction décimale (tab. 1). Elle est caractérisée par $D_{1}=10 \mathrm{~s}$ et par le fait que $48 \mathrm{~s}$ sont nécessaires pour obtenir réellement 4 réductions décimales. Swartling et Lindgren (1968) avaient observé une courbe de survie rectiligne, avec $\mathrm{D}=5,7 \mathrm{~s}$. 
b) Courbes de survie des autres souches

Selon les souches, la durée des traitements était de 3 ou de $10 \mathrm{mn}$. Trois types de courbes ont été observés :

\section{TABLEAU 1}

Paramètres caractéristiques des courbes de survie des spores de Bacillus dans l'eau oxygénée à $150 \mathrm{~g} / \mathrm{l}$, à $\mathrm{pH} 2$, à $80^{\circ} \mathrm{C}$

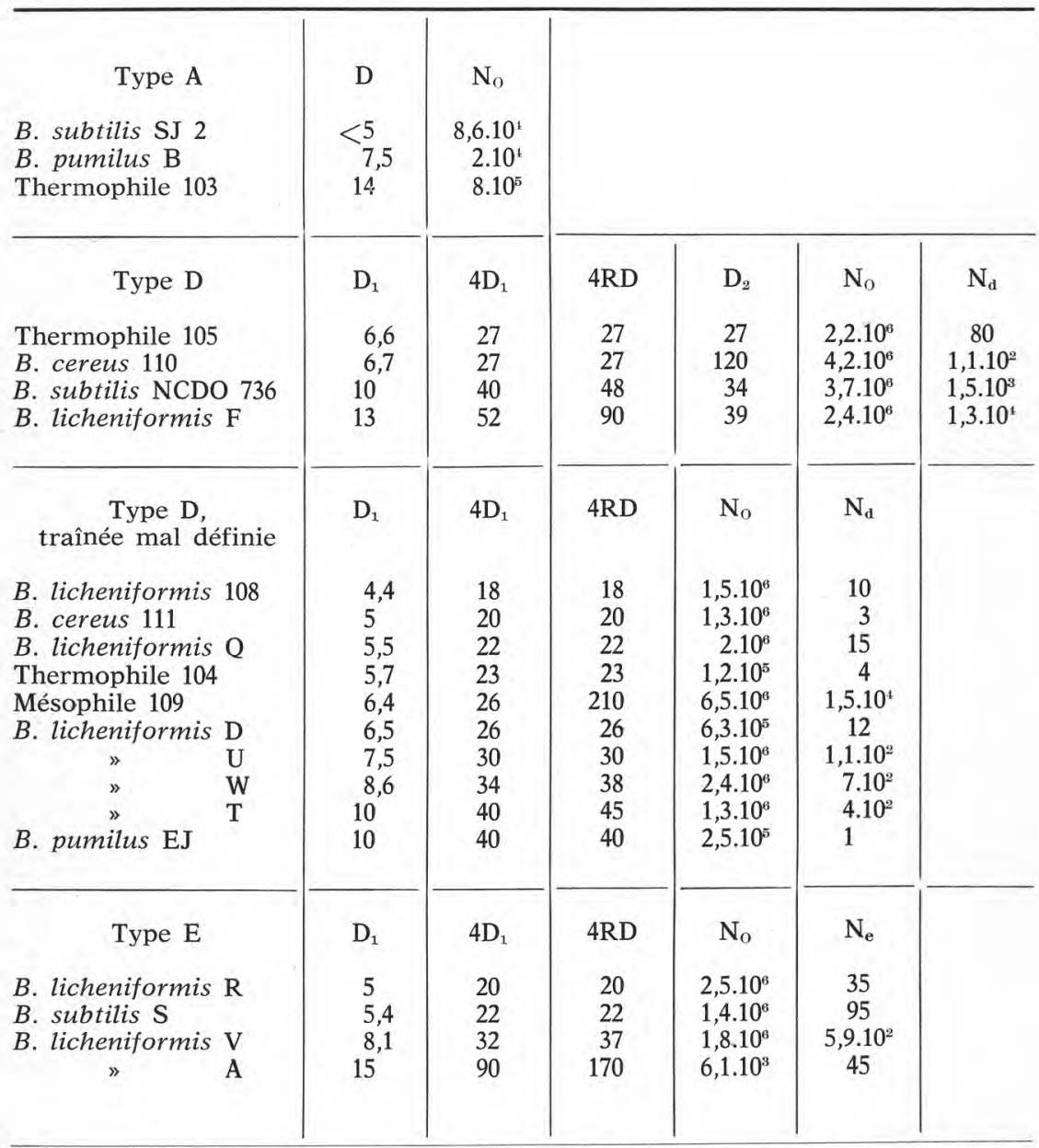

L'unité de temps est la seconde. Pour toutes les souches de types D et $E, t_{d}$ et $t_{e}$ sont très voisins de $30 \mathrm{~s}$. Dans la troisième colonne sont indiqués les temps nécessaires pour obtenir réellement 4 réductions décimales. La signification des symboles apparaît sur les figures 1 et 2 . 
fig. $1 \mathrm{~A})$;

des courbes sensiblement linéaires (type A, par exemple

- des courbes montrant une traînée, c'est-à-dire une variation brutale de la vitesse de destruction, à partir d'un taux de destruction précis, mais différant d'une souche à l'autre (type $\mathrm{D}$, par exemple fig. 1 D) ;
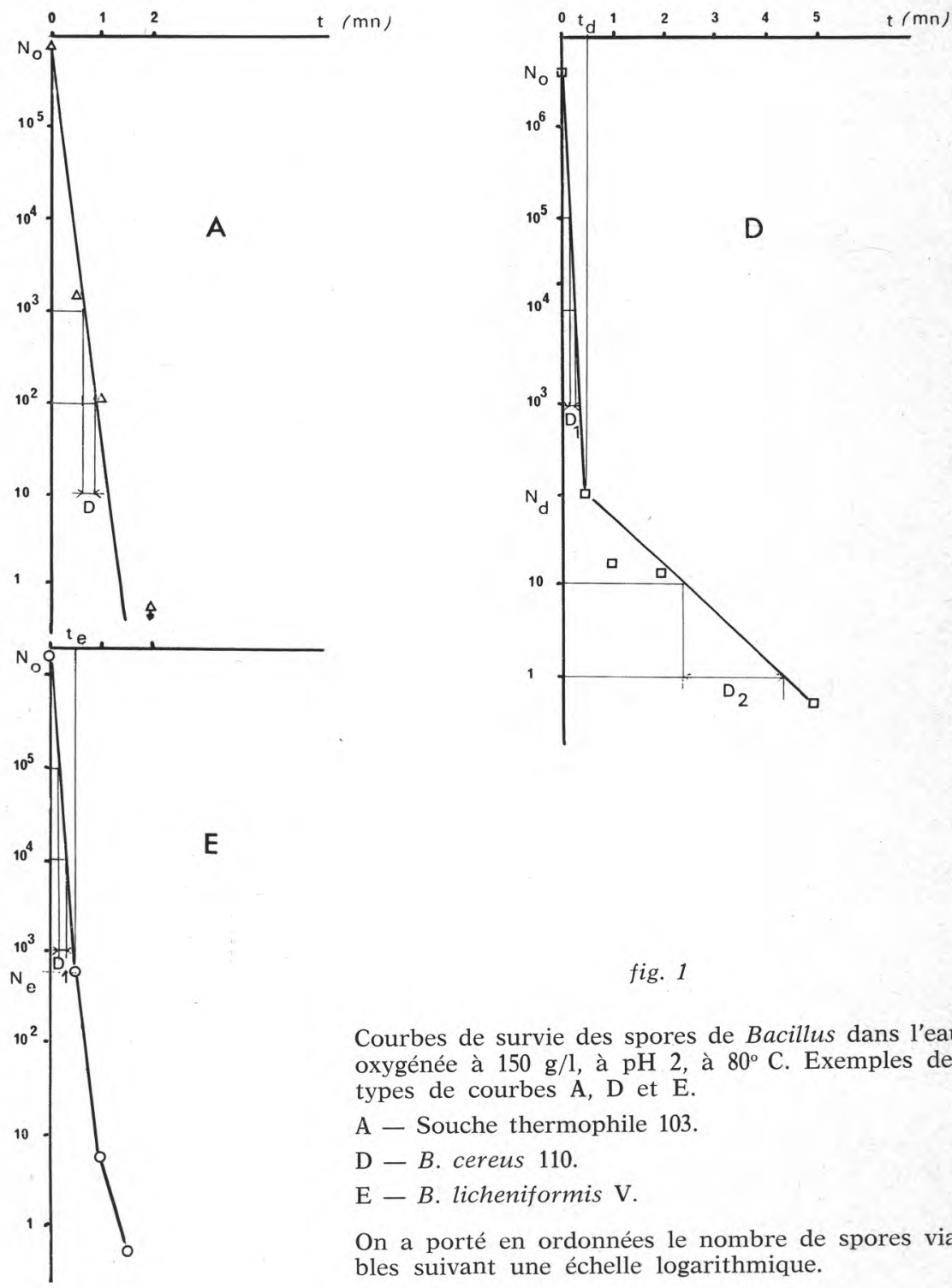

fig. 1

Courbes de survie des spores de Bacillus dans l'eau oxygénée à $150 \mathrm{~g} / \mathrm{l}$, à $\mathrm{pH} 2$, à $80^{\circ} \mathrm{C}$. Exemples des types de courbes A, D et E.

A - Souche thermophile 103.

$\mathrm{D}-B$. cereus 110.

$\mathrm{E}-B$. licheniformis $\mathrm{V}$.

On a porté en ordonnées le nombre de spores viables suivant une échelle logarithmique. 
fig. $1 \mathrm{E})$.

- des courbes concaves vers le haut (type E, par exemple

Le tableau 1 donne la répartition des souches en fonction du type de courbes de survie.

c) Valeur des paramètres de résistance

Le tableau 1 indique, pour les 21 souches les valeurs des paramètres caractéristiques qui décrivent complètement chaque courbe de survie. On voit que les courbes présentant une traînée ou une concavité sont les plus nombreuses : 18 au total. On remarque que toutes les souches de $B$. licheniformis se rattachent à ce groupe, mais que pour d'autres espèces, il y a une répartition entre types de courbes.

Les valeurs de $\mathrm{D}$ et de $\mathrm{D}_{1}$ s'échelonnent de 4,4 à $15 \mathrm{~s}$, c'est-à-dire de 1 à 3 au moins, mais les écarts entre les temps nécessaires pour obtenir 4 réductions décimales sont nettement plus grands, puisqu'ils vont de $<20$ à $210 \mathrm{~s}$, c'est-à-dire de 1 à 10 au moins. Lorsque la valeur 4D est différente du temps réellement nécessaire pour obtenir 4 réductions décimales, c'est le signe que la traìnée ou la concavité commence entre la première et la quatrième réduction décimale. $\mathrm{Ce}$ cas se reproduit 7 fois.

La résistance des souches est sans liaison apparente avec l'espc̀ce à laquelle elles appartiennent, ni avec le caractère mésophile ou thermophile. Les valeurs de D présentées dans le tableau 1 sont également sans rapport avec l'existence de traînées ou de concavité.

2) Résistance en milieu acide, a $80^{\circ} \mathrm{C}$

Pour analyser l'effet du traitement par l'eau oxygénée à $\mathrm{pH} 2$ à $80^{\circ} \mathrm{C}$, il nous a semblé utile d'étudier l'effet du traitement à $80^{\circ} \mathrm{C}$ à $\mathrm{pH} 2$, en l'absence d'eau oxygénée.

a) Aspect des courbes de survie

L'aspect des courbes de survie des spores chauffées à $80^{\circ} \mathrm{C}$ en milieu acide est très varié. Nous avons observé les types de courbes suivants :

- des droites (type A, fig. 2 A);

- des droites précédées d'un épaulement (type B, fig. 2 B);

- des droites précédées par une concavité vers le haut ne se prolongeant pas sur plus d'une réduction décimale (type C, fig. 2 C) ;

- des courbes à traînées (type $\mathrm{D}$, fig. $2 \mathrm{D}$ ).

Le tableau 2 donne la répartition des souches en fonction du type de courbe de survie.

b) Valeur des paramètres de résistance

Le tableau 2 décrit l'ensemble des courbes de survie des souches. Celles-ci se répartissent entre les quatre types décrits par Pflug et 

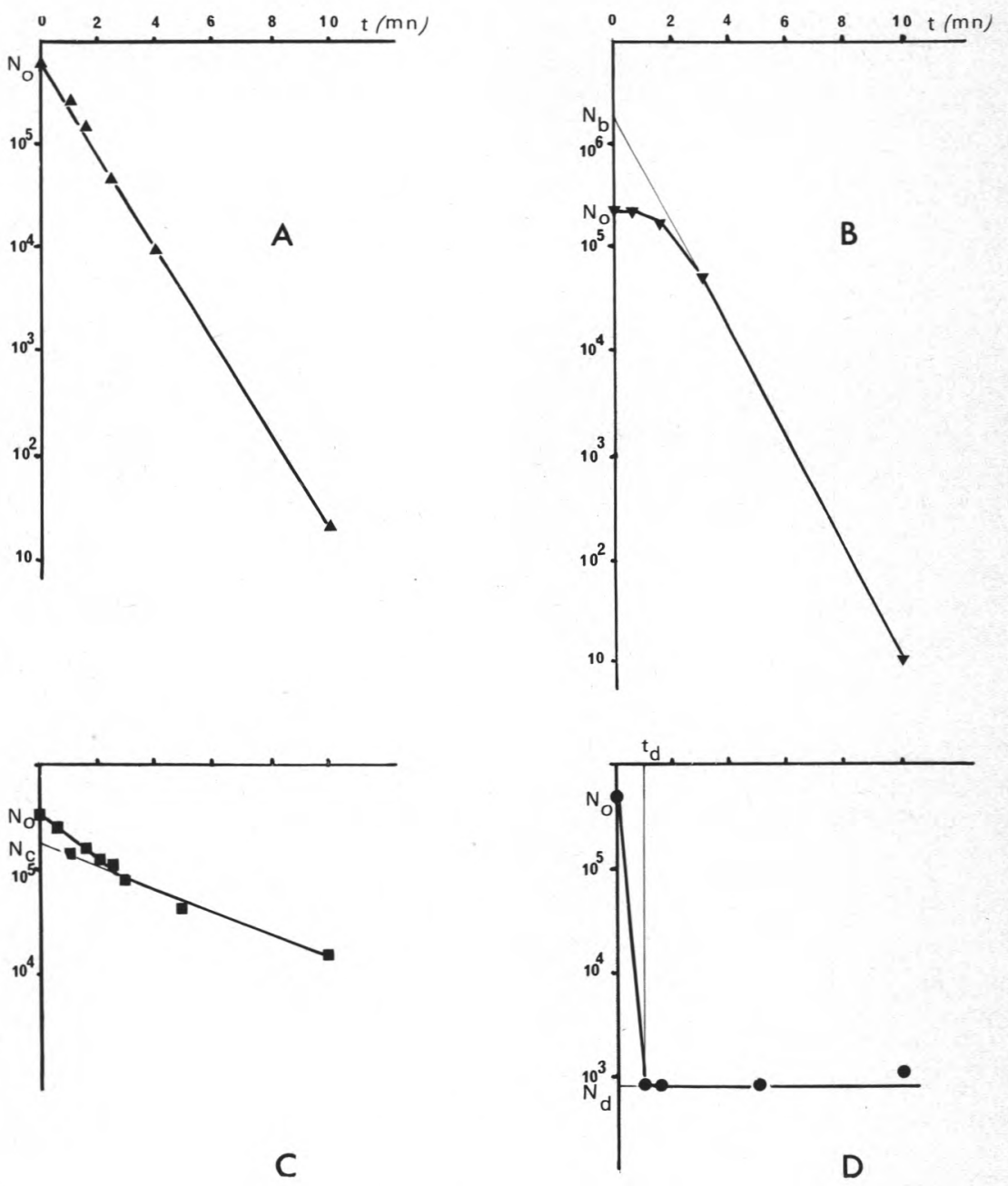

fig. 2

Courbes de survie des spores de Bacillus en milieu acide à $\mathrm{pH} 2$, à $80^{\circ} \mathrm{C}$. Exemples des types de courbes A, B, C et D.

A - B. licheniformis A.

B - B. pumilus EJ.

C - B. cereus 110 .

D - Souche thermophile 104.

On a porté en ordonnées le nombre de spores viables suivant une échelle logarithmique. 
Schmidt (1968). Cependant les courbes de types B et C, c'est-à-dire les courbes présentant une anomalie pendant la première réduction décimale, sont les plus nombreuses (15 souches). Les espèces sont réparties entre les différents types de courbes. On remarque que les courbes à épaulement (type $\mathrm{B}$ ) ont toutes un $\mathrm{D}$ inférieur à 7,7 mn, mais que 5 des 6 souches dont la courbe de survie appartient au

\section{TABLEAU 2}

Paramètres caractéristiques des courbes de survie des spores de Bacillus dans un milieu acide à $\mathrm{pH} 2,0$, à $80^{\circ} \mathrm{C}$

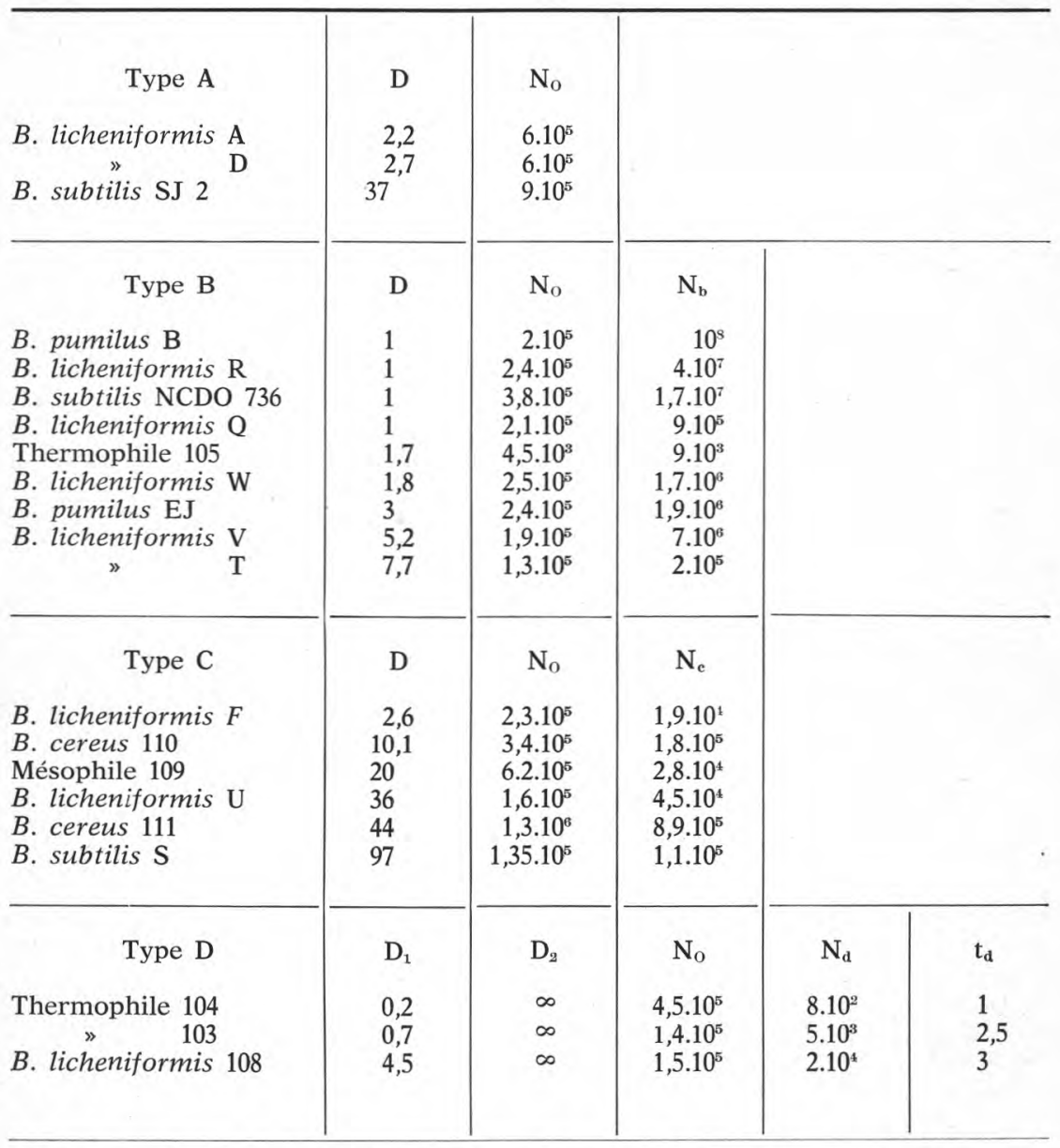

L'unité de temps est la minute. La signification des symboles apparaît sur les figures 1 et 2 . 
type $\mathrm{C}$ ont un $\mathrm{D}$ supérieur à $10 \mathrm{mn}$. On remarque également que, pour les courbes à traînées (type $\mathrm{D}$ ), $\mathrm{D}_{1}$ est petit (inférieur à $4,5 \mathrm{mn}$ ) mais que $\mathrm{D}_{2}$ est infini, c'est-à-dire qu'il n'y a aucune destruction pour une fraction de la population de spores. Les valeurs de $D$ et $D_{1}$ s'échelonnent de 0,2 à $97 \mathrm{mn}$, c'est-à-dire de 1 à 485, mais sans corrélation entre l'espèce et la résistance, ni entre la température optimale de croissance et la résistance.

\section{c) Comparaison avec le traitement en présence d'eau oxygénée}

Les courbes de survie des spores en milieu acide sont plus diversifiées que les courbes de survie en présence d'eau oxygénée. En effet, dans ce dernier cas, la destruction est plus rapide et l'aspect de la courbe au cours de la première réduction décimale du nombre de spores n'est pas connu avec précision : les particularités des types $\mathrm{B}$ et $\mathrm{C}$ n'apparaissent pas. Au contraire, en milieu acide, lorsque la destruction est lente, c'est l'apparition éventuelle de traînées ou de concavités vers le haut (types D et E) qui n'a pu être détectée, en raison de la durée limitée des temps de contact. Ceci explique peutêtre que si les souches présentent en majorité une courbe de survie à traînée ou à concavité (types $\mathrm{D}$ et $\mathrm{E}$ ) en présence d'eau oxygénée, c'est l'inverse que nous observons en milieu acide.

Sur les trois souches dont les courbes de survie ont une traînée en milieu acide, une seule présente une courbe sans traînée en présence d'eau oxygénée, la souche thermophile 103. La courbe de survie d'une seule souche, B. subtilis SJ 2, est du type A pour les deux traitements. Les deux autres souches qui ont une courbe normale en milieu acide ont respectivement une traînée et une concavité en présence d'eau oxygénée ( $B$. licheniformis $\mathrm{D}$ et $\mathrm{A})$. Il ne semble donc pas y avoir de rapport entre le type des courbes de survie à un traitement et celui des courbes lors de l'autre traitement.

La comparaison entre les tableaux 1 et 2 ne fait pas non plus apparaître la moindre corrélation entre d'une part la résistance en milieu acide et d'autre part la résistance en présence d'eau oxygénée. Telle souche pratiquement insensible à la seule acidité du milieu de traitement - par exemple B. subtilis SJ 2 - est rapidement détruite par addition d'eau oxygénée. Telle autre souche moyennement résistante en présence d'eau oxygénée - par exemple B. pumilus B - est parmi les souches les moins résistantes en milieu acide.

\section{3) Résistance dans l'eau oxygénée a $80^{\circ} \mathrm{C}$ en FonCtion du pH}

Deux souches ont été choisies pour cet essai :

- B. cereus 111, dont la résistance en milieu acide est parmi les plus élevées ( $\mathrm{D}=44 \mathrm{mn}$ ) et dont la courbe de survie en présence d'eau oxygénée présente une traînée, semble donc devoir être une bactérie " à problème "; 
TABLEAU 3

Paramètres caractéristiques des courbes de survie des spores de Bacillus dans l'eau oxygénée à $150 \mathrm{~g} / \mathrm{l}$, à $80^{\circ} \mathrm{C}$, en fonction du $\mathrm{pH}$.

\begin{tabular}{|c|c|c|c|c|c|c|c|c|}
\hline & $\mathrm{pH}$ & $\mathrm{D}_{1}$ & $4 \mathrm{D}_{1}$ & $4 \mathrm{RD}$ & $\mathrm{D}_{2}$ & $\mathrm{~N}_{0}$ & $\mathbf{N}_{\mathrm{d}}$ & $t_{d}$ \\
\hline B. cereus 111 & $\begin{array}{l}2,9 \\
4,6 \\
6,7 \\
7,7\end{array}$ & $\begin{array}{l}0,3 \\
0,17 \\
0,15 \\
0,19\end{array}$ & $\begin{array}{l}1,2 \\
0,68 \\
0,6 \\
0,76\end{array}$ & $\begin{array}{l}5,9 \\
0,68 \\
1,0 \\
2,3\end{array}$ & $\begin{array}{l}7,6 \\
1,8 \\
1,6 \\
0,9\end{array}$ & $\begin{array}{r}7.10^{5} \\
10^{6} \\
8,2.10^{5} \\
7.10^{5}\end{array}$ & $\begin{array}{r}5.10^{2} \\
10^{2} \\
1,7.10^{2} \\
7.10^{3}\end{array}$ & $\begin{array}{l}0,5 \\
0,5 \\
0,5 \\
0,5\end{array}$ \\
\hline B. subtilis SJ 2 & $\begin{array}{l}2,9 \\
4,6 \\
6,7 \\
7,7\end{array}$ & $\begin{array}{c}0,09 \\
0,13 \\
0,12 \\
0,2\end{array}$ & $\begin{array}{l}0,36 \\
0,52 \\
0,48 \\
0,8\end{array}$ & $\begin{array}{l}0,36 \\
0,9 \\
0,8 \\
1,0\end{array}$ & $\begin{array}{l}\overline{2,2} \\
1,4 \\
0,35\end{array}$ & $\begin{array}{l}7,5 \cdot 10^{5} \\
7,5 \cdot 10^{5} \\
7,5 \cdot 10^{5} \\
7,5 \cdot 10^{5}\end{array}$ & $\begin{array}{c}-\overline{1} \cdot 10^{2} \\
1,5 \cdot 10^{2} \\
2,7 \cdot 10^{3}\end{array}$ & $\begin{array}{l}\overline{0,5} \\
0,5 \\
0,5\end{array}$ \\
\hline
\end{tabular}

L'unité de temps est la minute.

Dans la quatrième colonne sont indiqués les temps nécessaires pour obtenir réellement 4 réductions décimales. La signification des symboles apparaît sur les figures 1 et 2 .

- B. subtilis SJ 2 qui, malgré une bonne résistance en milieu acide, est au contraire très facile à détruire par l'eau oxygénée.

Une troisième souche fait l'objet d'une autre publication.

Avec $B$. cereus le nombre de survivants se stabilise après cinq réductions décimales à $\mathrm{pH}$ 2,9 (tab. 3). A partir de $\mathrm{pH} \mathrm{4,6} \mathrm{et} \mathrm{au-dessus,}$ les courbes de survie présentent une traînée moins marquée. Di est maximal à $\mathrm{pH}$ 2,9 et sensiblement invariable aux autres $\mathrm{pH}$.

En ce qui concerne $B$. subtilis SJ 2, seule la courbe de survie à $\mathrm{pH} 2,9$ est rectiligne au moins pour les 6 premières réductions décimales. Si le $\mathrm{pH}$ semble exercer une influence sur les caractéristiques des traînées, il semble n'en exercer aucune sur la valeur de $D_{1}$, et ceci pour les deux souches. Aux $\mathrm{pH}$ supérieurs, à partir de la troisième réduction décimale, une traînée apparaît. Le tableau 3 montre que $\mathrm{D}_{1}$ est maximal à $\mathrm{pH} 7,7$ et minimal à $\mathrm{pH}$ 2,9.

Toutes les courbes de survie ont été établies avec un nombre de spores et une durée suffisante pour confirmer d'une manière sûre les traînées observées au cours des essais précédents. Malheureusement, les différences observées entre les deux souches empêchent toute conclusion précise quant à l'influence du $\mathrm{pH}$, en dehors du fait même que cette influence existe.

\section{4) Résistance dans L'EAU OXYGÉNÉE a $26^{\circ} \mathrm{C}$ EN FONCTION DU pH}

a) Aspect des courbes de survie

Les deux souches utilisées précédemment ont été traitées à $26^{\circ} \mathrm{C}$ dans l'eau oxygénée à $230 \mathrm{~g} / 1$ (tab. 4). Avec la souche B. subtilis SJ 2, 


\section{$T A B L E A U 4$}

Paramètres caractéristiques des courbes de survie des spores de Bacillus dans l'eau oxygénée à $230 \mathrm{~g} / \mathrm{l}$, à $26^{\circ} \mathrm{C}$, en fonction du $\mathrm{pH}$

\begin{tabular}{|c|c|c|c|c|c|c|c|c|c|}
\hline & $\mathrm{pH}$ & $\mathrm{D}_{1}$ & $4 D_{1}$ & $4 \mathrm{RD}$ & $\mathbf{D}_{2}$ & $\mathrm{~N}_{\mathrm{o}}$ & $\mathrm{N}_{\mathrm{b}}$ & $\mathrm{N}_{\mathrm{d}}$ & $t_{t}$ \\
\hline B. cereus 111 & $\begin{array}{l}2,9 \\
4,6 \\
6,7 \\
7,7\end{array}$ & $\begin{array}{r}2,3 \\
2,3 \\
<0,9 \\
2,7\end{array}$ & $\begin{array}{r}9,2 \\
9,2 \\
<3,6 \\
10,8\end{array}$ & $\begin{array}{r}>18 \\
>18 \\
>18 \\
11\end{array}$ & $\begin{array}{l}60 \\
19,5 \\
40 \\
5,2\end{array}$ & $\begin{array}{r}5.10^{7} \\
5.10^{7} \\
5.10^{7} \\
3,5.10^{7}\end{array}$ & $\begin{array}{l}- \\
- \\
-\end{array}$ & $\begin{array}{r}8.10^{3} \\
5.10^{3} \\
10^{4} \\
10^{4}\end{array}$ & $\begin{array}{r}9 \\
9 \\
<4 \\
9\end{array}$ \\
\hline$B$ subtilis $S J 2$ & $\begin{array}{l}2,9 \\
4,6 \\
6,7 \\
7,7\end{array}$ & $\begin{array}{l}4 \\
3,5 \\
3 \\
3\end{array}$ & $\begin{array}{l}16 \\
14 \\
12 \\
12\end{array}$ & $\begin{array}{l}40 \\
20 \\
17 \\
21\end{array}$ & $\frac{15}{-}$ & $\begin{array}{r}5.10^{\mathrm{s}} \\
7,5.10^{\mathrm{s}} \\
9.10^{8} \\
7.10^{7}\end{array}$ & $\begin{array}{l}3,5 \cdot 10^{9} \\
4,5 \cdot 10^{10} \\
1,2.10^{11} \\
3,5 \cdot 10^{9}\end{array}$ & $\frac{8.10^{3}}{-}$ & $\frac{45}{-}$ \\
\hline
\end{tabular}

L'unité de temps est la minute.

Dans la quatrième colonne sont indiqués les temps nécessaires pour obtenir réellement 4 réductions décimales. La signification des symboles apparaît sur les figures 1 et 2 .

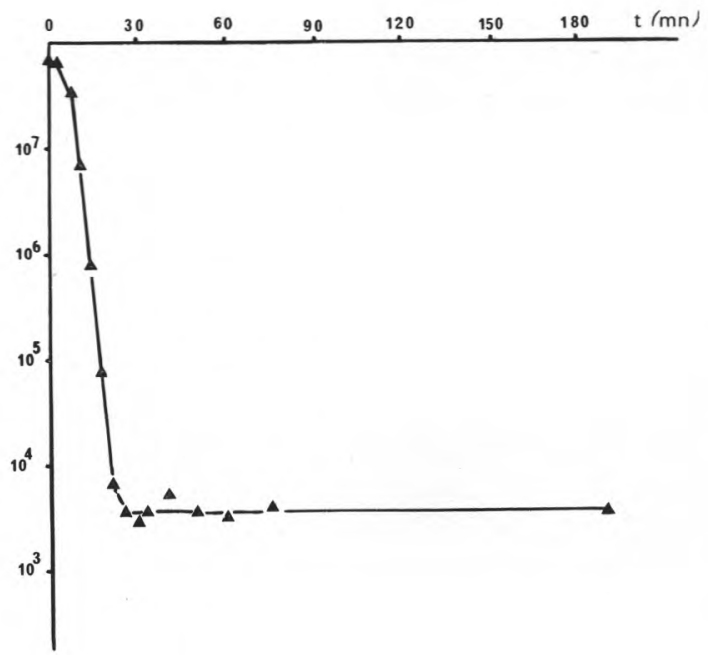

fig. 3

Courbe de survie des spores de B. subtilis SJ 2 dans l'eau oxygénée à $230 \mathrm{~g} / \mathrm{l}$ à $26^{\circ} \mathrm{C}$ à $\mathrm{pH} 7,7$ 
quel que soit le $\mathrm{pH}$, les courbes de survie ont un épaulement pendant les 10 premières minutes de contact, puis une décroissance rectiligne rapide à $\mathrm{pH} 4,6$ et 6,7. A pH 7,7 au contraire, la décroissance rectiligne ne se poursuit que sur quatre réductions décimales, puis cesse complètement : pendant les $3 \mathrm{~h}$ qu'a duré l'essai, le nombre de spores n'a plus varié (fig. 3). A pH 2,9 la courbe a le même aspect pendant les premières minutes, puis une traînée apparaît ; mais en $2 \mathrm{~h}$ il est possible de réaliser plus de six réductions décimales.

Avec B. cereus 111, en $9 \mathrm{mn}$, le nombre de spores décroît linéairement sans épaulement, puis il se stabilise après les quatre premières réductions décimales, et ceci à $\mathrm{pH} 2,9, \mathrm{pH} 4,6$ et $\mathrm{pH} 6,7$. A $\mathrm{pH}$ 7,7 le même phénomène apparaît mais la traînée est moins marquée (essais poursuivis respectivement 18 et $24 \mathrm{mn}$ ).

Le tableau 4 fait apparaître l'existence des épaulements et des traînées, et montre que le $\mathrm{pH}$ ne semble pas influer nettement sur la valeur de $D_{1}$ des spores dans l'eau oxygénée à température ambiante.

b) Comparaison entre l'aspect des courbes de survie en fonction du pH à 80 et à $26^{\circ} \mathrm{C}$

La comparaison entre les tableaux 3 et 4 ne montre pas de corrélation entre l'aspect des courbes d'une part et les valeurs de leurs paramètres caractéristiques d'autre part aux deux températures. A $80^{\circ} \mathrm{C}$, comme nous l'avons déjà remarqué, l'aspect du début des courbes reste hypothétique.

\section{Discussion}

En ce qui concerne le traitement par l'eau oxygénée à pH 2 à $80^{\circ} \mathrm{C}$, l'attention est dès l'abord attirée par le fait que les résultats de Swartling et Lindgren (1968) obtenus avec les spores de $B$. subtilis NCDO 736 n'ont pas été retrouvés. La courbe de survie présente une concavité vers le haut au lieu d'être rectiligne et la valeur de D (pour la partie rectiligne de la courbe) est presque le double de celle observée par les auteurs suédois.

Or le protocole expérimental utilisé ici diffère de celui de Swartling et Lindgren (1968) par au moins deux points :

- l'homogénéité du milieu de traitement a été améliorée grâce à une agitation permanente ;

- une solution tampon a été ajoutée à l'eau oxygénée pour stabiliser la valeur du $\mathrm{pH}$ à 2,0 .

Ces précautions supplémentaires ont dû contribuer à améliorer la précision des résultats obtenus.

D'autre part, les milieux de culture employés pour la production des spores et leur numération sont différents de ceux qu'ont em- 
ployés Swartling et Lindgren (1968). Cela aussi peut avoir contribué à la divergence observée quant à la résistance des spores de $B$. subtilis NCDO 736 à l'eau oxygénée.

Quoi qu'il en soit, les résultats concernant la résistance des spores de $B$. subtilis NCDO 736 donnent une image incomplète et insuffisante de la résistance des spores de Bacillus à l'eau oxygénée. La courbe de survie est bien linéaire (Curran et al., 1940 ; Swartling et Lindgren, 1968), mais seulement dans sa partie initiale, c'est-à-dire seulement jusqu'à la deuxième réduction décimale, pour certaines souches. Au-delà, l'aspect des courbes de survie est variable : on observe soit des concavités vers le haut, soit des traînées presque horizontales.

Des trois facteurs étudiés ici qui interviennent dans le traitement par l'eau oxygénée à $80^{\circ} \mathrm{C}$ à $\mathrm{pH} 2$, l'eau oxygénée est le principal et son action a des caractéristiques qui la distinguent nettement de l'action des autres facteurs. La température a un effet d'accélération sur cette action, comme le montrent la comparaison avec les résultats obtenus à $26^{\circ} \mathrm{C}$, et les valeurs du $\mathrm{Q}_{10}$ comprises entre 1,3 et 3 selon Curran et al. (1940) et Swartling et Lindgren (1968). Pour ce qui est de l'effet de l'acidité, le $\mathrm{pH}$ ne semble pas avoir d'influence déterminante, tout au moins sur la première partie des courbes de survie. King et Gould (1969) ont également observé que les spores de $B$. cereus préalablement mises en contact avec $\mathrm{HCl}$ à $\mathrm{pH} 3$ en urée $6 \mathrm{M}, 1 \mathrm{~h}$ à $37^{\circ} \mathrm{C}$, étaient inactivées par l'eau oxygénée de la même manière que des spores d'un lot témoin.

D'après Alderton, Thompson et Snell (1964), des spores natives placées à une température létale dans un milieu acide perdent progressivement une partie de leurs ions $\mathrm{Ca}^{++}$, ce qui s'accompagne d'une diminution progressive de leur thermo-résistance au cours du traitement ; la conséquence est que la courbe de survie est concave vers le bas. Ce type de courbe de survie n'a été observé que pour 9 des 21 souches chauffées à $80^{\circ} \mathrm{C}$ à $\mathrm{pH} 2$. On doit donc admettre que les conditions expérimentales n'étaient pas suffisantes chez les autres souches pour provoquer la libération des ions $\mathrm{Ca}^{++}$. En effet chez les souches de Bacillus dont la courbe de survie a une concavité vers le haut, il est exclu que la résistance de leurs spores diminue au cours du traitement. Cette absence de libération des ions $\mathrm{Ca}^{++}$ pourrait expliquer le peu d'influence du $\mathrm{pH}$ sur le traitement par l'eau oxygénée à $80^{\circ} \mathrm{C}$, mais King et Gould (1969) estiment que les ions $\mathrm{Ca}^{++}$ne sont pas en cause dans l'action de l'eau oxygénée. La diversité dans l'aspect des courbes de survie serait en faveur de l'hypothèse d'un ou plusieurs sites sensibles (selon la souche) sur lequel (ou lesquels) l'agent létal agirait à des vitesses différentes (Prokop et Humphrey, 1970 ; Moats, 1971).

En ce qui concerne les courbes de survie ayant une traînée, deux positions sont possibles. La première consiste à considérer ces traînées comme des artéfacts, comme le font par exemple Prokop et Humphrey (1970) qui écrivent " la numération des petits nombres 
de bactéries est caractérisée par une grande variabilité, et dans bien des cas les traînées décrites par certains auteurs sont probablement dues à des techniques de numération sélectives ". La deuxième consiste à admettre l'existence de ces traînées, en supposant que la population bactérienne contient des fractions d'inégales résistances. C'est ainsi que Moats, Dabbah et Edwards (1971) donnent plusieurs exemples de traînées du même type que celles observées avec $B$. subtilis SJ 2 (fig. 2). Brown et Melling (1971) font un excellent historique de cette discussion qui a commencé il y a au moins 50 ans. Avec le traitement par l'eau oxygénée à $80^{\circ} \mathrm{C}$, les traînées que Swartling et Lindgren (1968) ont pu observer "sont expliquées par la difficulté de dénombrer une ou moins de une spore par $\mathrm{ml}$ ". Par conséquent, " de telles valeurs sont laissées de côté pour le tracé des courbes ». En fait, les résultats exposés dans le présent article montrent que les traînées sont observables avec des concentrations en survivants égales ou supérieures à $10 \mathrm{par} \mathrm{ml}$, et que les courbes de survie à concavité vers le haut sont mises en évidence avec des concentrations en survivants bien plus élevées. En conséquence il faut admettre l'existence des traînées en ce qui concerne le traitement par l'eau oxygénée ou le traitement acide.

Si l'on admet que les traînées sont expliquées par un manque d'homogénéité de la population de spores, l'intersection de la traînée extrapolée et de l'axe des ordonnées indique la proportion des spores les plus résistantes. Pour les courbes observées au cours de ce travail, il ne semble pas que, d'un mode de traitement à l'autre pour une même souche, l'extrapolation puisse donner le même résultat. Dans ce cas, Roberts et Hitchins (1969) suggèrent que le constituant de la spore qui est inactivé existe sous plusieurs formes interconvertibles : nous pourrions avoir ce phénomène dans le cas où l'on fait varier le $\mathrm{pH}$ du traitement, et ceci expliquerait peut-être pourquoi la traînée n'apparaît qu'à certains $\mathrm{pH}$. Quelle qu'en soit l'explication, ce phénomène des traînées, ou tout au moins celui de la concavité des courbes de survie vers le haut, est fréquemment noté lorsque l'on fait agir des produits chimiques sur les spores. Citons des traînées de B. subtilis et Bacillus A et B dans l'oxyde d'éthylène (Kristensen, 1970), et la concavité des courbes de survie des spores de $B$. subtilis var. niger dans l'hypochlorite de sodium (Jones, Hoffmann et Phillips, 1968). Dans le cas de l'influence du $\mathrm{pH}$ sur la résistance à l'eau oxygénée, nous pouvons dire que ces traînées apparaissent lorsque l'on s'écarte des conditions optimales pour la destruction, encore que ces conditions soient différentes d'une souche à l'autre. Une telle remarque peut également être faite à propos de la destruction des spores de B. subtilis par l'hypochlorite de sodium (Cousins et Allan, 1967). Enfin, des courbes de survie sigmoïdes ont été observées dans certains cas par Staat (1970) avec des spores de $B$. subtilis var. niger obtenues à différents $\mathrm{pH}$ et 
traitées par la chaleur sèche, sans que cela puisse être attribué à une hétérogénéité génétique de la population de spores.

Nous devons maintenant en venir aux implications technologiques, en tenant compte de la diversité des paramètres des courbes de survie, et pour certaines d'entre elles, de l'existence des traînées. Il existe plusieurs modes de calcul pour la détermination des temps de traitement :

a) La méthode habituelle consiste à prendre comme base de calcul les paramètres de résistance représentatifs de la moyenne de la population de spores. Elle est utilisée par exemple pour le calcul des traitements par la chaleur sèche, avec les paramètres de $B$. subtilis var, niger ATCC 9372 (Bond, Favero, Petersen et Marshall, 1971).

b) Une méthode plus sévère choisit parmi les souches connues celle qui possède la résistance la plus élevée. C'est ainsi que l'on calcule les barèmes de traitement par la chaleur humide en vue de la destruction des spores botuliniques avec Clostridium botulinum 33 A (Stumbo, 1965).

Ces deux méthodes utilisent les paramètres de courbes de survie rectilignes. Elles peuvent être mises en défaut par la présence de spores dont la courbe de survie présente une traînée (Bond et al., 1970 et 1971). D'où :

c) La méthode la plus sévère ne tient compte que des paramètres de la souche la plus résistante connue, même si sa courbe de survie a une traînée.

d) Une méthode qui est peut-être plus réaliste tient compte non pas d'une souche de référence ni d'une moyenne, mais de l'ensemble d'une population de souches jugée comme représentative (Bond et al., 1971).

Grâce à la figure 4, il est possible de déterminer le temps de contact nécessaire avec l'eau oxygénée en fonction du nombre de réductions décimales désirées. Trois courbes sont données dans la figure 4, qui ont été établies à l'aide des trois modes de calcul a, $c$ et $\mathrm{d}$ :

a) à partir du temps de réduction décimal moyen calculé à partir du D ou $\mathrm{D}_{1}$ de chaque souche, soit $7,9 \mathrm{~s}$;

c) à partir de la courbe de survie de la souche la plus résistante (Bacillus mésophile 109) ;

d) à partir des paramètres de chaque souche pondérés par la fréquence de ces souches à l'intérieur de notre échantillon, que nous avons provisoirement estimé comme représentatif.

Si l'on se limite à quatre réductions décimales, comme l'ont suggéré Swartling et Lindgren (1968) pour la stérilisation du matériau d'emballage, on obtient selon le mode de calcul adopté les trois valeurs : 32,210 ou $60 \mathrm{~s}$, valeurs qui sont supérieures à celles données par ces auteurs à partir de la courbe de survie de B. subtilis NCDO 736 limitée à sa partie rectiligne. Du fait de l'existence de traînées, les temps de contact augmentent très rapidement si l'on 
choisit un nombre de réductions décimales plıs élevé. Par exemple pour six réductions décimales on obtient d'après la figure 4 : $48 \mathrm{~s}, 6 \mathrm{mn}$ ou $4,4 \mathrm{mn}$.

L'étude de l'action de l'eau oxygénée à la température ambiante n'a été faite qu'à titre de comparaison avec l'action à haute température. Les résultats limités ainsi obtenus ne permettent pas d'éta-

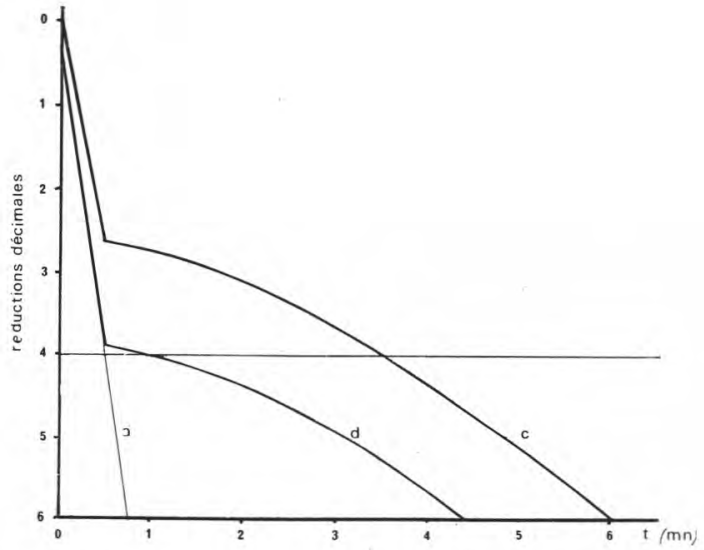

fig. 4

Calcul du temps de traitement par l'eau oxygénée à $150 \mathrm{~g} / \mathrm{l}$, à $\mathrm{pH} 2$, à $80^{\circ} \mathrm{C}$.

a : Courbe tracée à partir du temps de réduction décimale moyen.

c : Courbe tracée à partir de la courbe de survie de la souche la plus résistante.

$\mathrm{d}$ : Courbe tenant compte de la résistance de chaque souche, et de leur fréquence respective.

blir la valeur des temps de ontact avec la même rigueur. Indiquons simplement à titre de comparaison que les temps de contact pour obtenir quatre réductions décimales seraient supérieurs à $11 \mathrm{mn}$.

Les temps de contact que nous avons calculés sont sans commune mesure avec ceux qui sont utilisés dans la pratique pour le conditionnement du lait U.H.T. C'est qu'en réalité chaque appareillage met en œuvre un autre procédé stérilisant complémentaire. Dans certains appareillages le matériau d'emballage est préstérilisé par l'oxyde d'éthylène (Anonyme, 1969), et son temps de contact avec l'air ambiant au moment du chargement de la machine est réduit au minimum. Dans d'autres, le traitement par l'eau oxygénée 
est suivi par un véritable traitement thermique (Swartling et Lindgren, 1962) : les spores qui ont survécu à l'eau oxygénée sont amenées à l'état humide lors du passage dans le bain de la solution traitante et sont donc plus sensibles à la chaleur. En outre cette dernière décompose l'eau oxygénée restée sur le matériau, selon une réaction exothermique qui libère de l'oxygène, transitoirement à l'état moléculaire. L'effet destructeur de cet oxygène actif est peutêtre pour partie, à supposer qu'il existe réellement, dans l'efficacité stérilisatrice des appareillages où les traces d'eau oxygénée sont chassées par un courant d'air chaud.

Swartling et Lindgren (1962) ont donné quelques chiffres concernant l'effet complémentaire de la chaleur, mais aucune étude détaillée n'a été publiée à notre connaissance. Dans les appareillages où le matériau d'emballage traverse un bain de liquide aseptisant additionné d'un mouillant, il faut tenir compte en outre d'un effet de lavage mécanique.

\section{Remerciements}

Nous remercions $M$. R. L'Haridon des Etablissements E.P. Rémy et Cie, pour sa participation à ce travail.

Nous remercions $M$. G. Mocquot pour ses suggestions et ses critiques au cours de la rédaction de ce manuscrit.

\section{Abstract}

\section{Diversity of Bacillus spore resistance to hydrogen peroxide}

In general survivor curves of mesophilic and thermophilic Bacillus spores in 15 p. 100 hydrogen peroxide at $80^{\circ} \mathrm{C}$ (i) $\mathrm{pH} 2$ (21 strains) and (ii) $\mathrm{pH} 2.9$ to 7.7 (2 strains) are not logarithmic. Similar results were found (iii) in 23 p. 100 hydrogen peroxide at $26^{\circ} \mathrm{C}, \mathrm{pH} 2.9$ to 7.7 (2 strains). Most of the curves are definitely concave upwards and, with some strains, tails markedly. Furthermore, in conditions (iii) a shoulder appears during the first minutes of treatment. In the first part of survivor curves, the sporicidal action of hydrogen peroxide seems to be independent of $\mathrm{pH}$. Moreover, the spores of the 21 strains have 4 survivor curve types at $80^{\circ} \mathrm{C}, \mathrm{pH} 2$, indicating at least 4 inactivation mechanisms. Mean decimal reduction time (D) of the spore population, calculated for the first seconds of treatment (i), is $7.9 \mathrm{sec}$. But $3.5 \mathrm{~min}$. are required to obtain 4 effective decimal reductions of the most resistant strain in the same conditions. A curve is presented which includes each strain, with its own resistance and frequency, in the sample population. It shows that $1 \mathrm{~min}$. is required to obtain 4 decimal reductions of the population, and $4.4 \mathrm{~min}$. to obtain 6 decimal reductions. 


\section{$R$ é $s$ u é}

Les courbes de survie de spores de Bacillus mésophiles et thermophiles dans l'eau oxygénée à $15 \mathrm{p}$. 100 à $80^{\circ} \mathrm{C}$ (a) à $\mathrm{pH} 2$ (21 souches) ou (b) de pH 2,9 à 7,7 (2 souches) ne sont en général pas logarithmiques. Des résultats semblables ont été trouvés avec (c) de l'eau oxygénée à $23 \mathrm{p} .100$ à $26^{\circ} \mathrm{C}$, de $\mathrm{pH} 2,9$ à 7,7 (2 souches). La plupart des courbes sont nettement concaves vers le haut et montrent pour certaines souches des traînées marquées. En outre à la température la plus basse (c) un épaulement peut apparaître pendant les premières minutes de contact. L'action sporicide de l'eau oxygénée semble être indépendante du $\mathrm{pH}$ dans la première partie de la courbe de survie. Par ailleurs, les spores des 21 souches ont des courbes de survie très diverses en milieu acide à $\mathrm{pH} 2$, à $80^{\circ} \mathrm{C}$, impliquant qu'il existe au moins quatre mécanismes différents de résistance dans ces conditions. Le temps de réduction décimale (D) moyen de la population de spores, pour les 30 premières secondes de traitement dans les conditions (a) est de 7,9 s. Mais 3,5 mn sont cependant nécessaires pour obtenir 4 réductions décimales effectives de la souche la plus résistante dans les mêmes conditions. Une courbe est présentée qui tient compte de la fréquence des souches dans la population échantillon et de leur résistance respective. Elle montre que $1 \mathrm{mn}$ est nécessaire pour réduire le nombre de spores de la population par un facteur de $10^{4}$, et $4,4 \mathrm{mn}$ pour la réduire par un facteur de $10^{6}$.

Reçu pour publication en septembre 1971.

\section{Bibliographie}

Alderton (G.), Thompson (P.A.) and Snell (N.) (1964). - Heat adaptation and ion exchange in Bacillus megaterium spores. Science, 143, 141-143.

ANonyme (1969). - Valley Stream Farms in Jacksonville installs new longlife aseptic packaging system. Southern Dairy Products $J$., 84 (5), 36, 38-40.

AsHTon (T.R.) (1971). - Aseptic packaging, handling and transportation. Internat. Dairy Fed. Seminar, Malmö. Rep. 3.

Bond (W. W.), Favero (M. S.), Petersen (N. J.) and Marshall (J. H.) (1970). Dry-heat inactivation kinetics of naturally occuring spore populations. Appl. Microbiol., 20, 573-578.

Bond (W. W.), Favero (M. S.), Petersen (N. J.) and Marshall (J. H.) (1971). Relative frequency distribution of $D_{125 e}$ values for spore isolates from the Mariner - Mars 1969 spacecraft. Appl. Microbiol., 21, 832-836.

BRown (M. R. W.) and Melling (J.) (1971). - Inhibition and destruction of microorganisms by heat. In HuGo (W. B.) Inhibition and destruction of the microbial cell. Academic Press, Londres et New-York, 1-37.

Cousins (C. M.) and AllaN (C.D.) (1967). - Sporicidal properties of some halogens. J. Appl. Bacteriol., 30, 168-174. 
Curran (H. R.), Evans (F. R.) and Leviton (A.) (1940). - The sporicidal action of hydrogen peroxide and the use of crystalline catalase to dissipate residual peroxide. J. Bacteriol., 40, 423-434.

Hsu (D.S.) (1970). - Ultra-high temperature (U.H.T.) processing and aseptic packaging (A. P.) of dairy products. Damana Tech. Inc., New-York.

Jones (L. A.) Jr., Hoffman (R. K.) and Phillips (C. R.) (1968). - Sporicidal activity of sodium hypochlorite at subzero temperatures. Appl. Microbiol., 16, 787-791.

KING (W. L.) and Gould (G.W.) (1969). - Lysis of bacterial spores with hydrogen peroxide. J. Appl. Bacteriol., 32, 481-490.

KRISTENSEN (H.) (1970). - Ethylene oxide resistance of microorganisms in dust compared with the resistance of Bacillus subtilis spores. Acta Pathol. Microbiol. Scand. B, 78, 298-304.

LUBIENIECK vON SchelboRN (M.) (1970). - Aseptisches Abpacken und Abfüllen von Lebensmitteln. Verpackungs-Rundschau, 21, 51-54.

Lück (H.) (1956). - The use of hydrogen peroxide as a dairy preservative. Dairy Sci. Abstr., 18, 363-386.

Moats (W. A.) (1971). - Kinetics of thermal death of bacteria. J. Bacteriol., 105, 165-171.

Moats (W. A.), Dabbah (R.) and Edwards (V.M.) (1971). - Interpretation of non-logarithmic survivor curves of heated bacteria. J. Food Sci, 36, 523-526.

Pflug (I. J.) and Schmidt (C. F.) (1968). - Thermal destruction of microorganisms. In LAWRENCE (C. A.) and Block (S. S.). Disinfection, sterilization, and preservation. Lea and Febiger, Philadelphie, 63-105.

Prokop (A.) and HuMPhrey (A. E.) (1970). - Kinetics of disinfection. In Benarde (M. A.) Disinfection. Marcel Dekker Inc., New-York, 61-83.

RoberTs (T. A.) and Hitchins (A.D.) (1969). - Resistance of spores. In Gould (G. W.) and Hurst (A.). The bacterial spore. Academic Press, Londres et New-York, 611-670.

Russel (A.D.) (1971). - The destruction of bacterial spores. In Hugo (W. B.). - Inhibition and destruction of the microbial cell. Academic Press, Londres et New-York, 451-612.

Smith (N. R.), Gordon (R. E.) and Clark (F. E.) (1952). - Aerobic spore forming bacteria. U.S. Department of Agriculture, U.S. Government Printing Office, Washington, Agricult. Monograph $\mathrm{n}^{\circ} 16$.

StaAT (R. H.) (1970). - Logarithmic and non-logarithmic dry-heat survivor curves for Bacillus subtilis var. niger spores. Bact. Proceed., A 76.

Stumbo (C. R.) (1965). - Thermobacteriology in food processing. Academic Press, Londres et New-York.

Swartling (P.) and Lindgren (B.) (1962). - Aseptic filling in Tetra Pak. Sterilization of the paper. Alnarp Milk Dairy Res. Rep. $\mathrm{n}^{\circ} 66$.

Swartling (P.) and Lindgren (B.) (1968). - The sterilizing effect against Bacillus subtilis spores of hydrogen peroxide at different temperatures and concentrations. J. Dairy Res., 35, 423-428.

WANG (D. I. C.), Scharer (J.) and Humphrey (A. E.) (1964). - Kinetics of death of bacterial spores at elevated temperatures. Appl. Microbiol., 12, 451-454. 